\title{
Handwritten Electric Circuit Diagram Recognition: An Approach Based on Finite State Machine
}

\author{
Lakshman Naika R, Dinesh R, and Prabhanjan S
}

\begin{abstract}
In this paper we propose a method for recognizing hand drawn electronic circuit diagrams. The proposed method first detect and classify each components present in the hand drawn circuit diagram. For the purpose of component recognition, we have constructed the feature vector by combining Local Binary Pattern (LBP) and statistical features based on pixel density. Classification of components is done by using support vector machine (SVM) classifier. Upon detection and recognition of components, the proposed method subsequently uses the position and sequence of arrangement of components to determine the type of circuit. For the purpose of establishing the sequence of components we have used finite state machine. The proposed method represents the sequence of recognized components as a string. This string representation of circuit is fed to a Finite State Machine (FSM) to detect type of circuit. The proposed method has been tested on about 100 hand written circuit diagrams of varying complexities and of different types. The proposed component detection method gives over $99 \%$ accuracy whereas, the circuit recognition method has recognition rate of over $85 \%$ recognition rate for the circuit type recognition.
\end{abstract}

Index Terms - Circuit recognition, finite state machine, Local binary pattern, SVM, statistical features.

\section{INTRODUCTION}

Hand-drawn sketch is a natural and direct way to express ones thought and meaning and it is commonly used means in many fields. Diagram and sketches are widely used in engineering and architecture fields. This is mainly due to fact that a sketch is a convenient tool to catch rough idea, so that the designers can focus more on the critical issues rather than on the intricate details [1]. Recognition of electronic components and type of circuit from the handwritten circuit diagram is gaining importance. Electronic circuit diagram recognition has many applications like simulating the electronic characteristics of the circuit, layout planning for actual circuit design rendering, Human-computer interface for circuit analysis etc. Handwritten electronic circuit recognition also has significant application in teaching and conducting electronics subject. Where, the students were asked to write the circuit and to simulate its behaviors without actual circuit building. The current work aims mainly for developing a tool to evaluate the electronics circuit lab exercise. Usually, in electronics circuit lab / practical exam, student were asked to draw a particular circuit and get it reviewed by examiner and upon confirmation from the

Manuscript received October 6, 2018; revised April 17, 2019.

Lakshman Naika R and Dinesh R are with Jain University, Bangalore, Karnataka (State), India (e-mail: laxubdt@gmail.com, dr.dinesh@gmail.com).

Prabhanjan S i s with Jyoti Institute of Technology, Bangalore, Karnataka (State), India (e-mail: prabhanjan_us@gmail.com). examiner for the correctness of circuit, student is expected to build the circuit and conduct the experiments. This work minimizes the above process, where handwritten circuit will be fed to the proposed model and model will check for the validity of the circuit. Further, the proposed tool is also able to assist users to conduct experiment in simulation mode without actually building physical circuit.

While, handwritten text and symbol recognition is very well established research area where many researchers attempted to build text recognition system to recognize the characters for various languages. However, there are few works reported for recognizing the electronic components from the scanned electronic circuit diagram, but very few works are reported in literature to recognize the circuit type. In the next sub section we present the brief overview the existing work.

\section{RELATED WORK}

To automate the process of recognition of electric circuit from hand drawn circuit diagram, many attempts have been made in literature. In [2] hand written circuit schematic each component is segmented into linear sequence of image feature with conditional probability and components are recognized using HMM model. Whereas in [3] online hand drawn circuit diagram connected components and connector are segmented using a continuous time varying stroke stream in different eight directions using HMM and classification is performed using viterbi algorithm. [4] Address the problem of recognizing the circuits from the scanned image. In this method, image is pre-processed and components are segmented using spatial pixel density by manually choosing appropriate threshold. The components are recognized using statistical classifier using 35 different features from $\mathrm{Hu}$ moment, geometric measurement, histogram vector and polygonal approximation. The method has been tested on 9 components, the node are classified using syntactic analysis. However, the methods [3] and [4] do not handle the scale and orientation changes in the images. To address the problem of similarity transformation, [5] the method which works for online hand drawn circuit is recognized with scale, translation and rotation invariant recognition. The method decomposes each stroke into line and arc primitives. The system uses a structural and topological relation matching for recognition. In [6] this approach concentrated on circuit, text and figure. Each component associated with component label and values are recognized using any appropriate character recognition approach. Whereas the components are recognized by collection of horizontal, vertical and diagonal lines and are subsequently, clustered together based on production rule. After recognizing each component and its 
label construct the netlist of components and their connection. [7] On the other hand propose the method to recognize the digital logic circuit diagram comprising AND, OR and NOT gates. All the logical circuit symbols having one or more loop structures. The symbol recognition is using a decision-tree classifier. However, this method is capable of recognizing the components and circuits, it lags scalability with complex circuits with various electronic components. [8] Compares the performance of different techniques such as template matching, chain vector and Hough transform. The components are classified using neural network. [9] Segments the components using topology based technique from circuit and features are extracted using the Fourier descriptors and they have reported that they achieved the recognition accuracy of $90 \%$. [10] Addresses the recognition problem between different component like DC power supply and capacitor also similarity between roughly drawn wire and resistor by considering length and slope of stroke. Whereas [11] addresses the recognition of analog and digital components based on loop and non loop symbols using syntax-based and statistical classifier. In [12] HMM model is used to segment the components, it allows symbol hypothesis generation using 2D dynamic programming and components can be classified using neural network back propagation algorithm. [13], [14] Have proposed a method in which the objects are recognized based on its physical appearance by considering length breadth and connecting leads of components.

From afore discussion it is clear that quite a few methods are published in literature for hand drawn electric component and circuit recognition. Most of the method proposed in literature only focus on the problem of recognizing only components. Only few methods address the problem of both components and circuit recognition. It is also evident from the discussion that, many methods which address the problem of circuit recognition are online methods, where special device like stylus and tablet is required to draw a circuit. Such online method has limited application owing to special hardware requirement. Very few methods in literature address the problem of offline component and circuit recognition from hand-drawn sketches. The main limitation of such approaches is that either they are computationally very expensive or they work only on very limited components and circuit types lacking the scalability. Also, the recognition accuracy of such methods is very low limiting their application in real field. Hence, there is a need for developing an offline hand-drawn circuit and component recognition method which is computationally efficient and producing high recognition accuracy.

In view of this, in this paper, we propose a method for recognizing electronics components and circuits from the hand-drawn sketches. The proposed method is an offline method, means the method work on scanned image or the image captured from the hand held device like camera. Hence, unlike online methods the proposed method does not require any additional special hardware. The proposed method preprocesses the image before subjecting the image for component recognition. Component recognition is done by augmenting the features derived by component statistics and LBP [15]. Components are segmented from the circuit images using component density and morphological operations. Component recognition is performed by training the SVM classifier on the extracted features. Finally, to recognize the type of circuit (serial, parallel, LCR etc) the proposed method has used a finite state machine. The method represents the recognized components and their sequence in form of a string. This string is given as a input to the finite state machine which will classify the string as a particular type of circuit. Rest of the paper is organized as follows; In Section III we presents the details of the proposed method, where as experimental results are tabulated in Section V and Section VI describes the results analysis and finally paper concludes with the conclusion in Section VI.

\section{PROPOSED METHOD}

This section presents details of the proposed method. Fig. 1 gives the overall architecture of the proposed method.

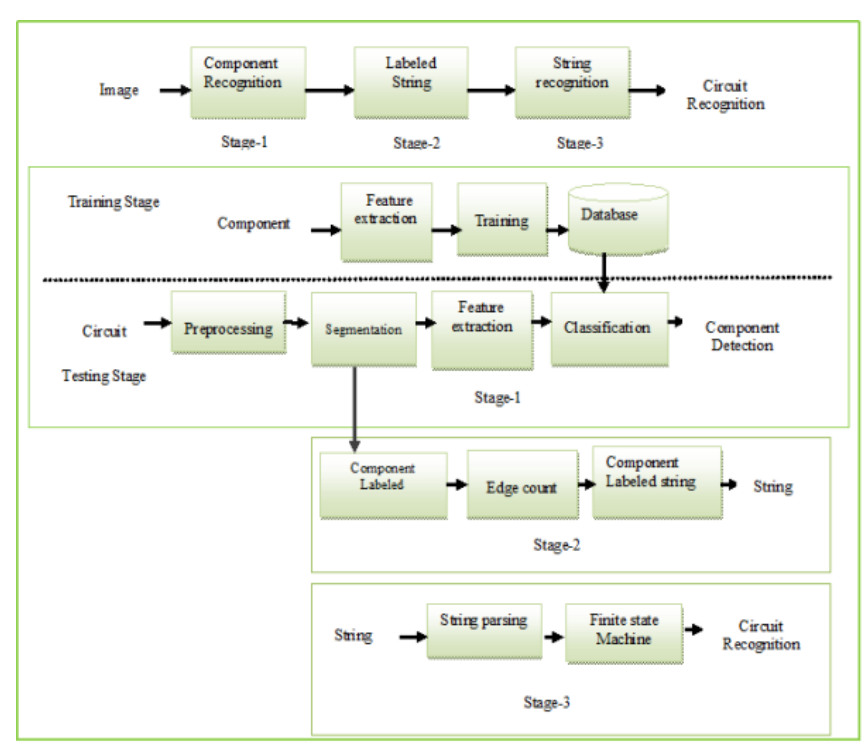

Fig. 1. Proposed architecture.

\section{COMPONENT RECOGNITION}

The proposed component recognition has two major stages (i) training stage and (ii) testing stage. In the training we train all possible components that need to be recognized using a SVM classifier. Whereas testing stage deals with assigning component class label for each component images posed as input.

\section{A. Training Phase}

For the purpose of training the classifier, we had considered 1000 hand-drawn electronics components. These component images are gathered from 9 different classes. It is necessary to represent the images in terms of its attribute or features which are numeric in nature to training the SVM classifier. In order to represent the training images in terms of its feature value, the proposed method uses feature descriptor derived by Local Binary Pattern (LBP) and five statistical features derived using pixel density statistics. The LBP feature is extracted from gray image and statistical features are from binary image. For LBP feature extraction the colour image converted into gray image, the salt and pepper noise is removed by averaging $M \times M$ neighbourhood pixel and replaced centre pixel in the output image. The morphological operation like dilation and erosion is applied to remove the discontinuity and gaps between lines. Thereafter applied to LBP feature extraction, the LBP feature extraction method is 
first introduced by Ojala et al. [15]. Local Binary Pattern is describing the texture details of a surface. The texture patterns are summarized into a histogram. The histogram values converted into single feature vector. The LBP operator works in a $3 \times 3$ pixel block of image, the central pixel of block adjusted to a threshold, the texture details can be obtain based on sign difference between centre pixel and its neighbourhood pixels. For each pixel in the image, the binary value is obtained by thresholding neighbour pixel with central pixel. The neighbour pixel become one, if the compared value greater than or equal to threshold otherwise zero. Fig. 2 pictorially describe the LBP operation. The operator uses in two patterns one is uniform and one more is non uniform, the uniform pattern has number of bitwise transition from 0 to 1 or vice versa is at most 2 . For example 01110000 ( 2 transitions) and 11001111 (2 transitions) are uniform, where as pattern has more than 2 transition, such pattern called non-uniform pattern example 11001001 (4 transitions) and 01010011 (6 transitions). The occurrence of output label in non-uniform pattern is single label only where as in uniform pattern have 58 labels. In this work, we have considered an image of size $48 \times 48$. The LBP features extracted in a two level in the first level 59 features from whole image and second level the image divided into 8 cells horizontally and 8 cells vertically, each cell with $6 \times 6$ pixels, totally 64 cells are generated, again extract 59 features from each cell, totally it generates $59+64 \times 59=3835$ features. For statistical feature the input component image converted to gray images, the gray images bearing large variation in intensity. These variations are poses a challenge on recognition. For operational convinces image has to change bi-level intensity. The gray image is converted into a binary level by adjusting threshold using Otsu's approach [16]. The salt and pepper noise is removed by averaging $M \times M$ neighbourhood pixel and replaced centre pixel in the output image. The morphological operation like dilation and erosion is applied to remove the discontinuity and gaps between lines. The binary image is inverted, background to black and foreground to object for operational convenience.

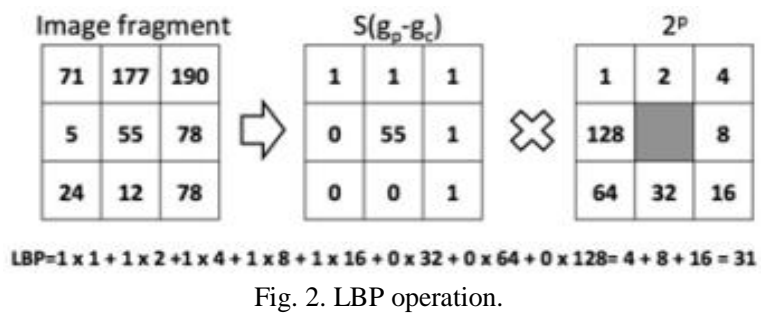

Further to improve the component recognition accuracy, the proposed method extracts additional features based on pixel density statistics. Statistical features are extracted by creating virtual Zoning on the component image. The statistical features viz, Euler number, Eccentricity, and Extent and orientation are extracted. In Zoning method the whole image divided into small zones, one feature value from each zone by considering number of 1's out of total element, in the Euler method by choosing number of connected component region minus number of holes present in the same connected component region, from Eccentricity method it create minimum bounding ellipse, major axis and minor axis, and calculate aspect ratio. The Extent is a ratio of number of pixel in the component region to number of pixel in a bounding box. The orientation is calculated by finding angle between $\mathrm{X}$-axis to the major axis of ellipse.

By augmenting LBP features and Statistical feature, the proposed method forms a feature vector. The dimension of the feature vector is 3949 . These feature vectors are used to train the classifier to classify the component image

SVM classifier is used to recognize / classify the giving component image. A Support Vector Machine (SVM) is a discriminative classifier formally defined by a separating hyperplane. In other words, given labeled training data (supervised learning), the algorithm outputs an optimal hyperplane which categorizes new examples. In which sense is the hyperplane obtained optimal? Let's consider the following simple problem:

For a linearly separable set of 2D-points which belong to one of two classes, find a separating straight line In Fig. 3, you can see that there exist multiple lines that offer a solution to the problem. Is any of them better than the others? We can intuitively define a criterion to estimate the worth of the lines.

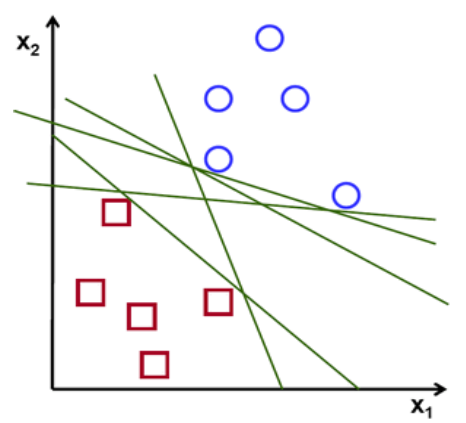

Fig. 3. Samples in feature space and possible hyper planes.

A line is bad if it passes too close to the points because it will be noise sensitive and it will not generalize correctly. Therefore, our goal should be to find the line passing as far as possible from all points as shown in Fig. 4.

Then, the operation of the SVM algorithm is based on finding the hyperplane that gives the largest minimum distance to the training examples. Twice, this distance receives the important name of margin within SVM's theory. Therefore, the optimal separating hyperplane maximizes the margin of the training data

For the purpose of training the component images, we have considered about 1000 images from nine different classes. Each class has about 100 images in the training set. We trained the SVM classifier by considering $80 \%$ of images for training and $20 \%$ for validation using 10 fold cross validation algorithm.

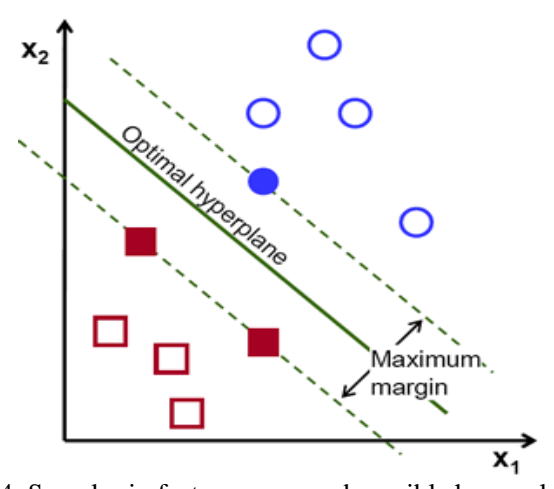

Fig. 4. Samples in feature space and possible hyper planes. 


\section{B. Testing Phase}

Once the SVM classifier is trained, we tested the trained SVM model on large set of images about 1000 component images. These testing images were not presented to the SVM classifier during the training. The proposed SVM classifier was able to recognize the component with recognition accuracy of over $99 \%$ for the testing images. This testifies that the built SVM classifier is not overfit and it has generalized for the purpose of component recognition. The details of the experimental results are presented in Section IV.

\section{Circuit ReCOGNITION}

In this phase hand drawn circuit input image is a given as a input for the proposed circuit recognition algorithm. Typical hand drawn circuit diagram is as shown in Fig. 5.

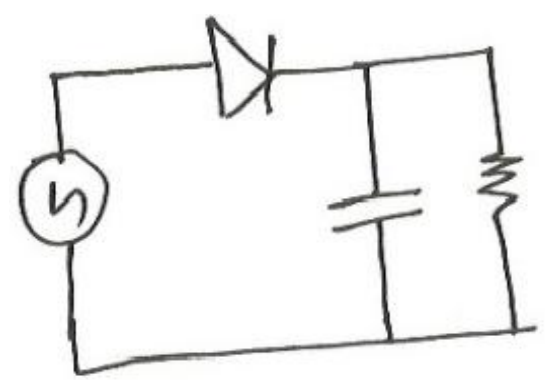

Fig. 5. Typical Hand drawn circuit diagram.

In order to recognize the circuit it is essential to first recognize the components present the electric circuit. The proposed method performs sequence of operations to recognize the hand drawn electric circuit. First, to remove the noise present in the captured image, noise removal filters are applied. Subsequently, component segmentation is performed to segment and localize the electrical components in the circuit diagram.

In the segmentation stage components are separated from circuit by studying the spatial density of a foreground and background pixel of the binarized image. The circuit line thickness is adjusted to single pixel level using some morphological operation. [4] The line and components have a different pixel density over a $K \times K$ neighbourhood region the pixel density of component is usually higher than that of the line segment over the same region $(K \times K)$. The pixel density is estimated over a $K \times K$ neighborhood region.

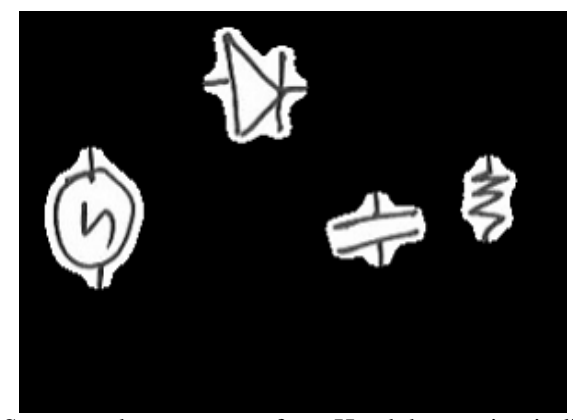

Fig. 6. Segmented components from Hand drawn circuit diagram.

In the proposed work threshold of pixel density is set to $T=3$ over $3 \times 3$ neighbored pixel. If the pixel density is less than threshold then the segment is considered as a line otherwise, it is consider as part of component. Upon finding components region, the region is further expanded using the region growing approach using the morphological operation. This process will separate the component from the circuit diagram as show in Fig. 6.

The segmented components region will be given as inputs to the SVM classifier which was already trained using the training images (as explained in previous sub section). The recognition label is recorded for further circuit recognition. For circuit recognition, it is also important to preserve the order of recognition of the components.

\section{A. Labeled String}

In the proposed method, the hand written circuit image is preprocessed. The circuit is connected by components, line and line connected edges. The components are segmented using the region grown approach using morphological operations. The recognition sequence of these components is also recorded to have complete information about the circuit. The recognized components are labeled in the same sequential order i.e. $\Sigma=\{C 1, C 2, C 3 \ldots . . C n\}$ where $C 1=$ component $1, C 2=$ component $2, C n=n^{\text {th }}$ components.

Each component labeled by $C 1=S, C 2=D, C 3=C$ and $C 4=R$ and so on forms a string sequence. The generated string sequence for the circuit shown in Fig. 5 is of the form $S 1=\{S$, $D, C, R\}$. After string formation, the system also, consider number of edges/ junctions present in the circuit. The number of junction information is used to classify a circuit as either serial circuit or parallel circuit.

The number of edges for above circuit is six (green color dots), the edges are shown in the Fig. 8. The number of junction count is important to identify the type of circuit (serial or parallel) as shown in Fig. 7. The edge count is represented by $S 2=\left\{N_{c}\right\}$ where c=count. The complete string represented by $S=S 1+S 2 \rightarrow S=\{S, D, C, R\}+\left\{N_{c}\right\}$. The string pattern for different circuit and its edge count is shown in the Table I.

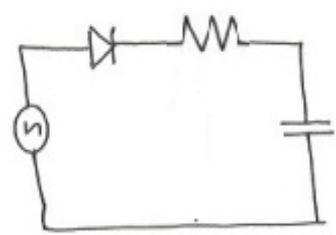

(a)

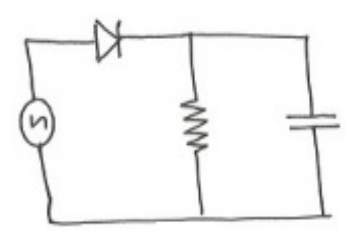

(b)
Fig. 7. (a) Series connection (b) parallel connection.

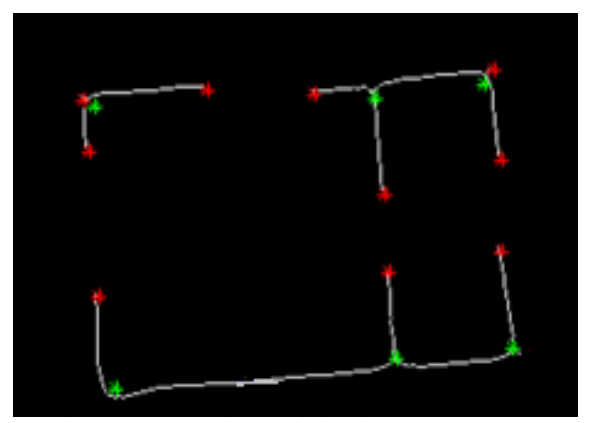

Fig. 8. Edge points of circuit (green color).

\section{B. Recognition}

The circuit recognition is very important step, the system uses the syntactic analysis to detect the circuit is based on 
grammar and string matching. A string is formed from sequence outcome of component generated by classifier and automata describe the string pattern for each circuit. The grammar is defined by five tuples

$$
G=\left\{Q, \Sigma, \partial, q_{0}, F\right\}
$$

where

$Q$ is a finite set of state

$\Sigma$ is a component of input symbol

$\Sigma \epsilon(C 1, C 2, C 3 \ldots C n)$

$C 1, C 2, \ldots, C n$ are electronics components i.e., diode, resister, transistor, capacitor etc.,

$\partial: Q \times \Sigma$ is the transition state

$q_{0} \epsilon Q$ is the initial state

$F$ is a final state

In the proposed method four hand drawn electronics circuits are chosen, i.e., fullwave rectifier, halfwave rectifier zener regulator and transistor amplifier as shown in Fig. 9. All the circuit connected by electronics components of Source $(S)$, Diode $(D)$, Transistor $(T)$, Resistance $(R)$, Capacitor $(c)$, Zener diode $(Z)$, The hand drawn circuit schematic first applied for preprocessing thereafter components separated from circuit by segmentation process. These separated components applied for feature extraction, based on features, the components are classified. These classified sequential outcomes of components are creating a string and also record the number of circuit edges. The fullwave rectifier and halfwave rectifier are circuits connected in two different fashions are shown in Fig. 9, both circuits gives same performance but these circuits generate different strings are shown in the Table I.

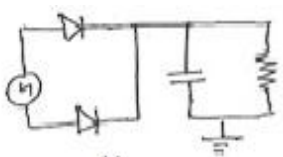

(a)

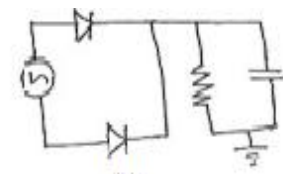

(b)

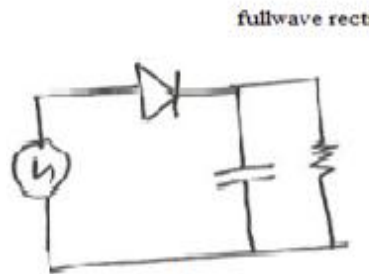

(c) (d)

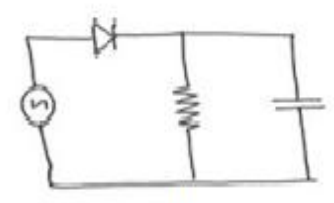

halfwave restifier

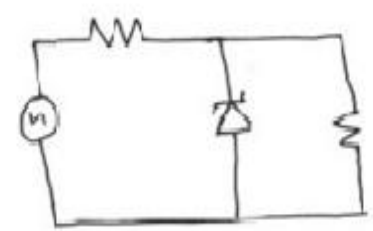

$$
\text { zener regulator }
$$

Fig. 9. Different Analog circuits considered for experimentation.

The half wave rectifier circuit connected by source, diode in series and resistance and capacitor are connected in parallel as shown Fig. 7(b), the string of this circuit is $\{S, D, R$, $C$ \}. The same components are connected in series as shown
Fig. 7(a), the string generated by this circuit is $\{S, D, R, C\}$ the both circuits are exhibits different performance but generates same string pattern. These circuits differentiated by edges of circuit, the series circuit have four edge points and the half wave rectifier circuit has the six edge points is shown in Fig. 8.

For the purpose of recognition of the generated string sequence for the given circuit, the proposed method has developed a finite state machine called finite automata.

TABLE I: STRING PATTERN COMBINATION

\begin{tabular}{|c|l|c|c|}
\hline sl.no & $\begin{array}{l}\text { Circuit type shown in } \\
\text { Fig. 8 }\end{array}$ & String pattren & $\begin{array}{l}\text { Number } \\
\text { of edge }\end{array}$ \\
\hline 1 & Fullwave circuit (a) & $(\mathrm{S}, \mathrm{D}, \mathrm{D}, \mathrm{C}, \mathrm{G}, \mathrm{R})$ & 8 \\
\hline 2 & Fullwave circuit (b) & $(\mathrm{S}, \mathrm{D}, \mathrm{D}, \mathrm{R}, \mathrm{G}, \mathrm{C})$ & 8 \\
\hline 3 & Halfwave rectifier (a) & $(\mathrm{S}, \mathrm{D}, \mathrm{C}, \mathrm{R})$ & 6 \\
\hline 4 & Halfwave rectifier (b) & $(\mathrm{S}, \mathrm{D}, \mathrm{R}, \mathrm{C})$ & 6 \\
\hline 5 & Zener regulator & $(\mathrm{S}, \mathrm{R}, \mathrm{Z}, \mathrm{R})$ & 6 \\
\hline 6 & Amplifier & $(\mathrm{S}, \mathrm{R}, \mathrm{R}, \mathrm{T}, \mathrm{R}, \mathrm{C})$ & 5 \\
\hline
\end{tabular}

A finite automaton is a popular method for syntactic recognition of patterns, [1] have used Finite Automata for detection of corner points. Fig. 10 shows the finite state machine of the proposed method for recognition of hand drawn circuit diagram.

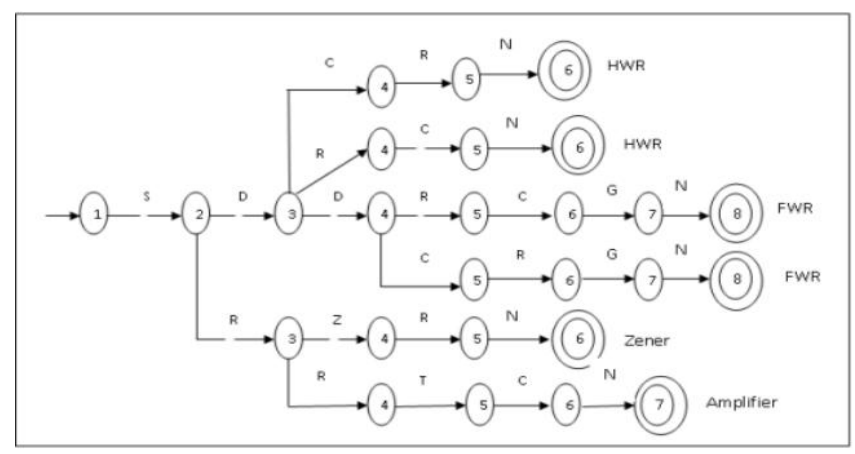

Fig. 10. Proposed Finite state model for circuit recognition.

The string sequence generated after recognition of components and components labeling, the string will be given as input to the finite state machine shown in Fig. 10. The finite state machine parses the string and makes appropriate transition to recognize the type of circuit.

Hence the details of the proposed algorithm for circuit recognition and component recognition can be summarized as below.

Algorithm: Hand drawn Electronic Circuit Recognition

Inputs: (i) Scanned / captured images of hand drawn circuit.

(ii) SVM model for component recognition (which was pre-trained as part of offline training process)

Output: Type of Circuit (Amplifier, Zener, Fullwave rectifier, halfwave rectifier, etc)

\section{Method:}

Step 1: Pre-process the image as explained in section 4A to remove the noise and to convert the input image into a binary image.

Step 2: Perform image segmentation to detect and localize the component region as explained in Section IVA using the approach of region growing using region analysis and morphological operations. 
Step 3: Crop the region around the component detected in Step 2 and give the cropped image region as input to Component Recognizer using SVM Classifier. This step will output the component label (type of component)

Step 4: Repeat Step 3 for all candidate component regions present in the image and collect the recognized labels and their sequence of recognition (left to right and top to bottom).

Step 5: From a string of labels obtained by Step 3 and Step 4 by concatenating the string label in sequence of their recognition.

Step 6: Find number of junction points in the hand drawn circuit to find whether given circuit is a parallel circuit or serial circuit, as explained in Section VB

Step 7: Give the string constructed in Step 5 to Finite State Machine defined in Section VB to find type of circuit.

Step 8: Output the circuit type.

End Hand drawn Electronic Circuit Recognition Algorithm

\section{EXPERIMENTAL RESULT}

In this section we present the details of experiments conducted to establish the superiority of the proposed method.

First to test the efficacy of the proposed component recognition algorithm, we tested the component recognition model with about 1000 images. The proposed method was able to recognize the components with recognition accuracy of over $99 \%$.

TABLE II: CONFUSION MATRIX OF COMPONENT RECOGNITION

\begin{tabular}{|c|c|c|c|c|c|c|c|c|c|}
\hline $\mathrm{D}$ & $\mathrm{T}$ & $\mathrm{Rh}$ & $\mathrm{S}$ & $\mathrm{Z}$ & $\mathrm{RV}$ & $\mathrm{G}$ & $\mathrm{Cv}$ & $\mathrm{Ch}$ & \\
\hline 92 & 0 & 0 & 0 & 0 & 0 & 1 & 0 & 1 & $\mathrm{D}$ \\
\hline 0 & 101 & 0 & 0 & 0 & 0 & 0 & 0 & 0 & $\mathrm{~T}$ \\
\hline 0 & 0 & 146 & 0 & 0 & 0 & 0 & 0 & 0 & $\mathrm{Rh}$ \\
\hline 0 & 0 & 0 & 88 & 1 & 0 & 0 & 0 & 0 & $\mathrm{~S}$ \\
\hline 1 & 0 & 0 & 1 & 96 & 2 & 0 & 0 & 0 & $\mathrm{Z}$ \\
\hline 0 & 0 & 1 & 0 & 1 & 119 & 0 & 0 & 0 & $\mathrm{Rv}$ \\
\hline 0 & 0 & 0 & 0 & 0 & 0 & 89 & 0 & 0 & $\mathrm{G}$ \\
\hline 0 & 0 & 0 & 0 & 0 & 0 & 1 & 136 & 0 & $\mathrm{CV}$ \\
\hline 0 & 0 & 0 & 0 & 0 & 0 & 0 & 0 & 137 & $\mathrm{Ch}$ \\
\hline
\end{tabular}

Table II presents the Confusion matrix of the proposed component recognition method. The components mentioned in the above table are $\mathrm{D}, \mathrm{T}, \mathrm{Rh}, \mathrm{S}, \mathrm{Z}, \mathrm{Rv}, \mathrm{G}, \mathrm{Cv}$ and $\mathrm{Ch}$ are represents diode, transistor, resistance with horizontal orientation, source, zener diode, resistance with vertical orientation, ground, capacitor with vertical and horizontal orientation. It is evident from the Table II that the proposed component recognition method was quite accurately recognize the components with $100 \%$ recognition rate for Transistors and $\mathrm{Cv}$ (capacitor with vertical orientation) and higher recognition even for other components. Further, Table IV presents the classification accuracy of the proposed method.

Further for the purpose of establishing the superiority of the proposed method in recognizing the circuit we performed experiments with various circuits with varying complexities.

Table III presents the strings generated by the proposed method for the different types of circuits shown in Fig. 8. For establishing the goodness of the proposed method is recognizing the components and generating the strings.

TABLE III: PATTREN StRING GENERATE OF CirCUITS SHOWn IN Fig. 9

\begin{tabular}{|l|l|l|l|}
\hline $\begin{array}{l}\text { S.n } \\
0\end{array}$ & $\begin{array}{l}\text { Circuit type shown in } \\
\text { figure \& }\end{array}$ & $\begin{array}{l}\text { String pattren } \\
\text { of edge }\end{array}$ \\
\hline 1 & Fullwave circuit (a) & $(\mathrm{S}, \mathrm{D}, \mathrm{D}, \mathrm{C}, \mathrm{G}, \mathrm{R})$ & 8 \\
\hline 2 & Fullwave circuit (b) & $(\mathrm{S}, \mathrm{D}, \mathrm{D}, \mathrm{R}, \mathrm{G}, \mathrm{C})$ & 8 \\
\hline 3 & Halfwave rectifier (a) & $(\mathrm{S}, \mathrm{D}, \mathrm{C}, \mathrm{R})$ & 6 \\
\hline 4 & Halfwave rectifier (b) & $(\mathrm{S}, \mathrm{D}, \mathrm{R}, \mathrm{C})$ & 6 \\
\hline 5 & Zener regulator & $(\mathrm{S}, \mathrm{R}, \mathrm{Z}, \mathrm{R})$ & 6 \\
\hline 6 & Amplifier & $(\mathrm{S}, \mathrm{R}, \mathrm{R}, \mathrm{T}, \mathrm{R}, \mathrm{C})$ & 5 \\
\hline
\end{tabular}

From all the above experiments, it is evident that the proposed method is quite accurate in recognizing the components and circuit types. Overall accuracy of the proposed method is over $99 \%$ and also the proposed method is very fast in recognition and hence suitable for real time applications.

TABLE IV: C LASSIFICATION ACCURACY OF THE PROPOSED METHOD FOR 9 DIFFERENT CLASSES

\begin{tabular}{|c|c|c|c|c|c|c|c|c|c|}
\hline Sl no & TP rate & FP rate & Precision & Recall & F-Measure & $\mathrm{MCC}$ & ROC Area & PRC Area & Class \\
\hline 1 & 0.979 & 0.001 & 0.989 & 0.979 & 0.984 & 00.982 & 0.989 & 0.970 & 1 \\
\hline 2 & 1.000 & 0.000 & 1.000 & 1.000 & 1.000 & 1.000 & 1.000 & 1.000 & 2 \\
\hline 3 & 1.000 & 0.001 & 0.993 & 1.000 & 0.996 & 0.996 & 0.999 & 0.993 & 3 \\
\hline 4 & 0.989 & 0.001 & 0.989 & 0.989 & 0.988 & 0.998 & 0.994 & 0.979 & 4 \\
\hline 5 & 0.960 & 0.002 & 0.980 & 0.960 & 0.970 & 0.966 & 0.979 & 0.944 & 5 \\
\hline 6 & 0.983 & 0.002 & 0.983 & 0.983 & 0.983 & 0.981 & 0.991 & 0.969 & 6 \\
\hline 7 & 1.000 & 0.002 & 0.978 & 1.000 & 0.989 & 0.988 & 0.999 & 0.978 & 7 \\
\hline 8 & 0.993 & 0.000 & 1.000 & 0.993 & 0.996 & 0.996 & 0.996 & 0.994 & 8 \\
\hline 9 & 1.000 & 0.000 & 1.000 & 0.993 & 0.996 & 0.996 & 0.999 & 0.993 & 9 \\
\hline Weighted Avg & 0.990 & 0.001 & 0.990 & 0.990 & 0.990 & 0.989 & 0.989 & 0.993 & \\
\hline
\end{tabular}

\section{CONCLUSION}

In this paper, we propose a method for recognizing the hand drawn circuit using the finite state machine. This paper also presented the details of the proposed component recognition algorithm which is based on the features derived based on LBP and Component density statistics and subsequently classified using SVM classifier. In order to corroborate the efficacy of the proposed method, we have conducted extensive experiments on the large number of images with varying degree of complexity. Based on the experimental results, it has been established that the proposed method is able to recognize the components and type of circuits with high degree of accuracy. Also the proposed method takes quite less time to recognize the components and type of circuit and hence suitable for the real time applications. 


\section{REFERENCES}

[1] R. Dinesh and D. S. Guru, "Finite automata inspired model for dominant point detection: A non-parametric approach," in Proc. International Conference on Computing: Theory and Applications (ICCTA 2007), March 2007, Kolkata, India.

[2] P. Salao, "A recognition system for symbols of electronics components in hand-written circuit diagrams," in Proc. the IEEE, pp. 257-286.

[3] Y. M. Zhang, C. Viard, and L. M. Wu, "An online hand drawn electric circuit diagram recognition system using hiden markov models," in Proc. IEEE International Symposium on Information Science and Engineering, 2008, pp. 143-147.

[4] B. Edward and V. Chandan, "Machine recognition of hand-drawn circuit diagrams," in Proc. 2000 IEEE International Conference on Acoustics, Speech and Signal Processing, Istanbul, Turkey, June 2000 no. 6, pp. 3618-3621.

[5] J. P Valois, M. C^ot'e, and M. Cheriet, "Online Recognition of Sketched Electrical Diagrams," in Proc. ICDAR, 2001, pp. 460-464.

[6] D. Bailey, A. Norman, and G. Moretti, Electronics Schematic Recognition, pp. 147-151.

[7] A. Okazaki, T. Kondo, K. Mori, S. Tsunekawa, and E. Kawamoto, "An automatic circuit diagram reader with loop- structure-based symbol recognition," IEEE Transactions on Pattern Analysis and Machine Intelligence, vol. 10, no. 3, May 1988.

[8] R. B. Johnson, "Recognizing electronics symbols using neural networks," The Institution of Electrical Engineering, Savoy London, UK, 1999.

[9] Y. Liu and Y. Xiao, Citcuit Sketch Recognition, Stanford University, Stanford.

[10] O. Ejofodomi and S. Ross, "Online handwritten circuit recognition on a tablet PC," in Proc. the $33^{\text {rd }}$ Applied Imagery Pattern Recognition Workshop, 2004.

[11] E. O. Jesus and R. A. Lotufo, "ECIR-An Electronic circuit diagram image recognizer," Anais SIBGRAPI, 1998.

[12] G. Feng, C. V. Gaudin, and Z. Sun, "On-line hand-drawn electric circuit diagram recognition using 2D dynamic programming," Pattern Recognition, vol. 42, pp. 3215-3223, 2009.

[13] M. K. chigateri and K. M. Manjuvani, "The detection of electrical and electronics components using $\mathrm{K}$ nearest neighbor classification algorithm," International Research Journal of Engineering and Technology, vol. 3, 2016.

[14] C. Alvarado, Multi-domain Hierarchical Fresh-Stroke Recognition Using Graphical Model, Verlag, London, 2011.
[15] T. Ojala, M. Pietikäinen, and D. Harwood, "A comparative study of texture measures with classification based on feature distributions," Pattern Recognit., vol. 29, no. 1, pp. 51-59, 1996.

[16] N. Otsu, "A threshold selection method from gray-level histograms," IEEE Transactions on Systems, Man, and Cybernetics, vol. 9, no. 1, pp. 62-66, 1979

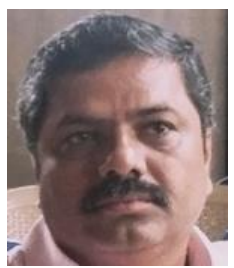

Lakshman Naika $\mathbf{R}$ is a research scholar in Jain University, Bangalore, Karnataka, India. $\mathrm{He}$ is working as an associate professor in UBDT College of Engineering, .Karnataka, India. He has more than 18 years' experience in teaching. He has completed B.E degree in electronics and communication from Mysore University, M.Tech degree in Electronics Engineering from Visvesvaray Technological University, Katnataka, India and pursuing $\mathrm{PhD}$ degree in Jain University. His research interest is image processing. He is a member of Indian Society for Technical Education and Institution of Electronics and Telecommunication engineers.

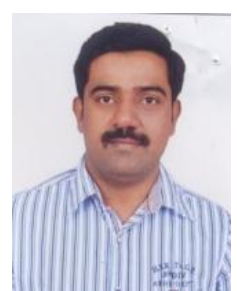

Dinesh $\mathbf{R}$ is a visiting professor in Jain University, Bangalore, Karnataka, India. He has over 16 years' teaching, research and industrial experience. $\mathrm{He}$ completed his M.Sc and $\mathrm{PhD}$ in computer science in 2001 and 2006, respectively, from University of Mysore. His research interest includes, image processing, machine learning, computer vision and industrial automation. He has successfully guided many research and industrial projects. Dinesh has over 100 publications and 28 patents to his credit.

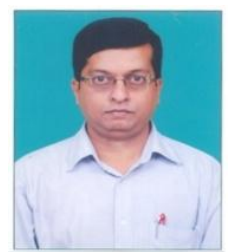

Prabhanjan $\mathbf{S}$ is a professor at Jyothy Institute of technology Bengaluru Karnataka, India. He has completed B.E, M.Tech and Ph.D in computer Science \& Engineering. He has around 22 years' experience in teaching. His area of research is image processing, pattern recognition, compiler design. 